\title{
THE WEYL CALCULUS AND CLIFFORD ANALYSIS
}

\author{
Brian JefFeries and Alan McIntosh
}

\begin{abstract}
The connection between Clifford analysis and the Weyl functional calculus for an $n$-tuple of bounded selfadjoint operators is noted. The operators do not necessarily commute with each other.
\end{abstract}

\section{INTRODUCTION.}

The spectral theorem for a selfadjoint operator $T$ acting on a Hilbert space $H$ facilitates the expression of a function $f(T)$ of $T$ in terms of an integral $f(T)=$ $\int_{\sigma(A)} f(\lambda) d P(\lambda)$ with respect to a spectral measure $P$. In general, no such representation is possible for an $n$-tuple $A=\left(A_{1}, \ldots, A_{n}\right)$ of non-commuting bounded selfadjoint operators acting on a Hilbert space $H$. Nevertheless, the Weyl functional calculus $f \mapsto f_{\mathrm{W}}(A)$ for $A$ is a means of constructing functions $f_{\mathrm{W}}(A)$ of the system $A$ of operators, for suitable smooth functions $f$ defined on $\mathbb{R}^{n}$. It was proposed by $\mathrm{H}$. Weyl for the pair $(P, Q)$ of unbounded self adjoint operators, where $P$ is the momentum operator and $Q$ is the position operator in quantum mechanics. For the case $n=2$, the function $\mathbf{x}_{1} \mathbf{x}_{2}:\left(x_{1}, x_{2}\right) \mapsto x_{1} x_{2}$, for all $x_{1}, x_{2} \in \mathbb{R}$, belongs to the domain of the Weyl functional calculus and $\left(\mathrm{x}_{1} \mathbf{x}_{2}\right)_{\mathrm{W}}(A)=\left(A_{1} A_{2}+A_{2} A_{1}\right) / 2$. In general, polynomials on $\mathbb{R}^{n}$ are mapped by the Weyl functional calculus into the corresponding polynomials in the $n$-tuple $A$ of operators, but with products suitably symmetrised.

A similar phenomenon emerges in Clifford analysis. A monogenic function $f$ defined on $\mathbb{R}^{n+1}$, and with values in a finite dimensional Clifford algebra, is a function lying in the kernel of the Dirac operator - a higher dimensional analogue of the Cauchy-Riemann equations. Every analytic function in $n$ real variables has a unique monogenic extension to $\mathbb{R}^{n+1}$. The monogenic extensions $\mathbf{z}_{1}$ and $\mathbf{z}_{2}$ of the functions $\mathbf{x}_{1}:\left(x_{1}, \ldots, x_{n}\right) \mapsto x_{1}$ and $\mathbf{x}_{2}:\left(x_{1}, \ldots, x_{n}\right) \mapsto x_{2}$, respectively, are easily written down. It turns out that the monogenic extension of the real valued function $x_{1} x_{2}$ to $\mathbb{R}^{n+1}$ is $\left(\mathbf{z}_{1} \mathbf{z}_{2}+\mathbf{z}_{2} \mathbf{z}_{1}\right) / 2$, and, in general, the monogenic extension of polynomials on $\mathbb{R}^{n}$ are the corresponding polynomials in the $n$-tuple $\left(\mathbf{z}_{1}, \ldots, \mathbf{z}_{n}\right)$ of monogenic functions, but with products suitably symmetrised. The purpose of the present note is to elaborate on this formal similarity between the Weyl and Clifford calculi.

Received 11th August, 1997

Copyright Clearance Centre, Inc. Serial-fee code: 0004-9729/98 \$A2.00+0.00. 
The present work may be formulated in terms of Hermitian operators acting on a Banach space in place of selfadjoint operators on a Hilbert space, along the lines of [1], or in terms of bounded operators on a Banach space satisfying an exponential growth condition, as in [7, Condition 8.1]. However, the essential features are already present in the selfadjoint case addressed here. The situation where no growth condition is assumed is discussed in another article [5] that builds on work of James Picton-Warlow.

At one point in Section 5, some facts concerning the integration of vector valued functions are needed. Suppose that $(\Sigma, \mathcal{S}, \mu)$ is a measure space and $E$ is a sequentially complete locally convex space. Let $f: \Sigma \rightarrow E$ be a function for which there exist $E$ valued $\mu$-integrable $\mathcal{S}$-simple functions $s_{n}, n=1,2, \ldots$ such that $s_{n} \rightarrow f \mu$-amost everywhere, and for every continuous seminorm $p$ on $E, \int_{\Sigma} p\left(s_{n}-s_{m}\right) d \mu \rightarrow 0$ as $n, m \rightarrow \infty$. Then the integral $\int_{A} f d \mu$ of $f$ with respect to $\mu$, over a set $A \in \mathcal{S}$, is defined to be the limit $\lim _{n \rightarrow \infty} \int_{A} s_{n} d \mu$. The limit is independent of the approximating sequence $s_{n}, n=1,2, \ldots$; such a function $f$ is said to be Bochner $\mu$-integrable. It follows immediately that for a continuous linear map $T: E \rightarrow F$ between sequentially complete locally convex spaces $E$ and $F$, if $f$ is Bochner $\mu$-integrable, then $T \circ f$ is Bochner $\mu$-integrable and $T\left(\int_{A} f d \mu\right)=\int_{A} T \circ f d \mu$ for all $A \in \mathcal{S}$. A bounded continuous function with values in a Fréchet space or $L F$-space is Bochner integrable with respect to any finite regular Borel measure.

The basic notions of Clifford algebras are outlined in Section 2. Notation concerning Banach modules and Banach module homomorphisms is introduced in Section 3. General information concerning Clifford analysis is presented in Section 4. The connection between the Weyl and the Clifford calculi appeals to Theorem 5.4 of Section 5, which is actually proved not just for the Weyl functional calculus, but for any operator valued distribution with compact support. In Section 6, we identify the complement of the support of the Weyl functional calculus for an $n$-tuple $A$ of selfadjoint operators with a certain 'resolvent set' where the Cauchy kernel for $A$ is monogenic. Similar ideas have been advanced by Kisil [6], but Example 6.3 shows that the domain of monogenicity of the Cauchy kernel for a pair $\mathbf{T}$ of Pauli matrices cannot be calculated in the fashion of [6, Definition 3.1], so [6, formulae (9), (10)] as they stand are incorrect with the spectrum $\sigma(\mathbf{T})$ defined in $[6$, Definition 3.1].

\section{Clifford algebras.}

Let $\mathbb{F}$ be either the field $\mathbb{R}$ of real numbers or the field $\mathbb{C}$ of complex numbers. The Clifford algebra $\mathbb{F}_{(n)}$ over $\mathbb{F}$ is a $2^{n}$-dimensional algebra with unit defined as follows. Given the standard basis vectors $e_{0}, e_{1}, \ldots, e_{n}$ of the vector space $\mathbb{F}^{n+1}$, the basis vectors $e_{S}$ of $\mathbb{F}_{(n)}$ are indexed by all finite subsets $S$ of $\{1,2, \ldots, n\}$. The basis 
vectors are determined by the following rules for multiplication on $\mathbb{F}_{(n)}$ :

$$
\begin{aligned}
e_{0}=1, & \\
e_{j}^{2}=-1, & \text { for } 1 \leqslant j \leqslant n \\
e_{j} e_{k}=-e_{k} e_{j}=e_{\{j, k\}}, & \text { for } 1 \leqslant j<k \leqslant n \\
e_{j_{1}} e_{j_{2}} \cdots e_{j_{s}}=e_{S}, & \text { if } 1 \leqslant j_{1}<j_{2}<\cdots<j_{s} \leqslant n \text { and } S=\left\{j_{1}, \ldots, j_{s}\right\} .
\end{aligned}
$$

Here the identifications $e_{0}=e_{\emptyset}$ and $e_{j}=e_{\{j\}}$ for $1 \leqslant j \leqslant n$ have been made.

The product of two elements $u=\sum_{S} u_{S} e_{S}, u_{S} \in \mathbb{F}$ and $v=\sum_{S} v_{S} e_{S}, v_{S} \in \mathbb{F}$ is $u v=\sum_{S, R} u_{S} v_{R} e_{S} e_{R}$. According to the rules for multiplication, $e_{S} e_{R}$ is \pm 1 times a basis vector of $\mathbb{F}_{(n)}$. The scalar part of $u=\sum_{S} u_{S} e_{S}, u_{S} \in \mathbb{F}$ is the term $u_{\emptyset}$, also denoted as $u_{0}$.

The Clifford algebras $\mathbb{R}_{(0)}, \mathbb{R}_{(1)}$ and $\mathbb{R}_{(2)}$ are the real numbers, the complex numbers and the quaternions, respectively.

The conjugate $\overline{e_{S}}$ of a basis element $e_{S}$ is defined so that $e_{S} \overline{e_{S}}=\overline{e_{S}} e_{S}=1$. Denote the complex conjugate of a number $c \in \mathbb{F}$ by $\bar{c}$. Then the operation of conjugation $u \mapsto \bar{u}$ defined by $\bar{u}=\sum_{S} \overline{u_{S}} \overline{e_{S}}$ for every $u=\sum_{S} u_{S} e_{S}, u_{S} \in \mathbb{F}$ is an involution of the Clifford algebra $\mathbb{F}_{(n)}$. Then $\bar{u} \bar{v}=\overline{v u}$ for all elements $u$ and $v$ of $\mathbb{F}_{(n)}$. An inner product is defined on $\mathbb{F}_{(n)}$ by the formula $(u, v)=[u \bar{v}]_{0}=\sum u_{S} \overline{v_{S}}$ for every $u=\sum_{S} u_{S} e_{S}$ and $v=\sum_{S} v_{S} e_{S}$ belonging to $\mathbb{F}_{(n)}$. The corresponding norm is written as $|\cdot|$.

Suppose that $m \leqslant n$ are positive integers. The vector space $\mathbb{R}^{m}$ is identified with a subspace of $\mathbb{F}_{(n)}$ by virtue of the embedding $\left(x_{1}, \ldots, x_{m}\right) \mapsto \sum_{j=1}^{m} x_{j} e_{j}$. On writing the coordinates of $x \in \mathbb{R}^{n+1}$ as $x=\left(x_{0}, x_{1}, \ldots, x_{n}\right)$, the space $\mathbb{R}^{n+1}$ is identified with a subspace of $\mathbb{F}_{(n)}$ with the embedding $\left(x_{0}, x_{1}, \ldots, x_{n}\right) \mapsto \sum_{j=0}^{n} x_{j} e_{j}$.

\section{BANACH MODULES.}

A Banach space $X$ with norm $\|\cdot\|$ over $\mathbb{F}$ with an operation of multiplication by elements of $\mathbb{F}_{(n)}$ turning it into a left module over $\mathbb{F}_{(n)}$ is called a (left) Banach module over $\mathbb{F}_{(n)}$, if there exists $C \geqslant 1$ such that

$$
\|u x\| \leqslant C|u|\|x\|
$$

for all $u \in \mathbb{F}_{(n)}$ and $x \in X$. A right Banach module has a similar definition. The expression Banach module means a left and right Banach module. The vector space of all continuous right module homomorphisms from a Banach module $X$ to a Banach 
module $Y$ is denoted by $\mathcal{L}_{(n)}(X, Y)$. Thus, a bounded linear map $A: X \rightarrow Y$ belongs to $\mathcal{L}_{(n)}(X, Y)$ if $(A x) u=A(x u)$ for all $x \in X$ and $u \in \mathbb{F}_{(n)}$ and $\mathcal{L}_{(n)}(X, Y)$ is a left Banach module. Both $\mathcal{L}_{(n)}(X, Y)$ and the space $\mathcal{L}(X, Y)$ of continuous linear operators from $X$ to $Y$ are considered as Banach spaces over $\mathbb{F}$ with the uniform operator norm $\|\cdot\|$.

The algebraic tensor product $X_{(n)}=X \otimes \mathbb{F}_{(n)}$ of a Banach space $X$ over $\mathbb{F}$ with $\mathbb{F}_{(n)}$ is a Banach module. Elements of $X_{(n)}$ may be viewed as finite sums $u=\sum_{S} x_{S} \otimes e_{S}$ of tensor products of elements $x_{S}$ of $X$ with basis vectors $e_{S}$ of $\mathbb{F}_{(n)}$. Multiplication in $X_{(n)}$ by elements $\lambda$ of the Clifford algebra $\mathbb{F}_{(n)}$ is defined by $u \lambda=\sum_{S} x_{S} \otimes\left(e_{S} \lambda\right)$ and $\lambda u=\sum_{S} x_{S} \otimes\left(\lambda e_{S}\right)$. The tensor product notation $x_{S} \otimes e_{S}$ is written simply as $x_{S} e_{S}$. The norm on $X_{(n)}$ is taken to be $\|u\|=\left(\sum_{S}\left\|x_{S}\right\|_{X}^{2}\right)^{1 / 2}$.

The analogous procedure applies to a locally convex space $E$ to define the module $E_{(n)}$ with its induced locally convex topology. If $E$ and $F$ are two locally convex spaces, then the spaces $(\mathcal{L}(E, F))_{(n)}$ and $\mathcal{L}_{(n)}\left(E_{(n)}, F_{(n)}\right)$ are identified by defining the operation of $T=\sum_{S} T_{S} e_{S}$ on $u=\sum_{S} u_{S} e_{S}$ as $T(u)=\sum_{S, S^{\prime}} T_{S}\left(u_{S^{\prime}}\right) e_{S^{\prime}} e_{S^{\prime}}$. In the case that $E$ and $F$ are equal to a Banach space $X$, the norm of $T$ is given by $\|T\|=$ $\left(\sum_{S}\left\|T_{S}\right\|_{\mathcal{C}(X)}^{2}\right)^{1 / 2}$. Given $x \in E$ and $\xi \in F^{\prime}$, the element $\langle T x, \xi\rangle \in \mathbb{F}_{(n)}$ is defined for each $T=\sum_{S} T_{S} e_{S}$ belonging to $\mathcal{L}_{(n)}\left(E_{(n)}, F_{(n)}\right)$ by $\langle T x, \xi\rangle=\sum_{S}\left\langle T_{S} x, \xi\right\rangle e_{S}$.

\section{Clifford analysis.}

What is usually called Clifford analysis is the study of functions of finitely many real variables, which take values in a Clifford algebra, and which satisfy higher dimensional analogues of the Cauchy-Riemann equations.

A function $f: \mathbb{R}^{n+1} \rightarrow \mathbb{F}_{(n)}$ has a unique representation $f=\sum_{S} f_{S} e_{S}$ in terms of $\mathbb{F}$-valued functions $f_{S}, S \subseteq\{1, \ldots, n\}$ in the sense that $f(x)=\sum_{S} f_{S}(x) e_{S}$ for all $x \in \mathbb{R}^{n+1}$. Then $f$ is continuous, differentiable and so on, in the normed space $\mathbb{F}_{(n)}$, if and only if for all finite subsets $S$ of $\{1, \ldots, n\}$, its scalar component functions $f_{S}$ have the corresponding property. Let $\partial_{j}$ be the operator of differentiation of a scalar function in the $j$ 'th coordinate in $\mathbb{R}^{n+1}$ - the coordinates of $x \in \mathbb{R}^{n+1}$ are written as $x=\left(x_{0}, x_{1}, \ldots, x_{n}\right)$. For a continuously differentiable function $f: \mathbb{R}^{n+1} \rightarrow \mathbb{F}_{(n)}$ with $f=\sum_{S} f_{S} e_{S}$, the function $D f$ is defined by setting $D f=\sum_{S}\left(\partial_{0} f_{S} e_{S}+\sum_{j=1}^{n} \partial_{j} f_{S} e_{j} e_{S}\right)$ and $f D$ is specified by $f D=\sum_{S}\left(\partial_{0} f_{S} e_{S}+\sum_{j=1}^{n} \partial_{j} f_{S} e_{S} e_{j}\right)$. 
Now suppose that $f$ is an $\mathbb{F}_{(n)}$-valued, continuously differentiable function defined in a neighbourhood of an open subset $U$ of $\mathbb{R}^{n+1}$. Then $f$ is said to be left monogenic in $U$ if $D f(x)=0$ for all $x \in U$ and right monogenic in $U$ if $f D(x)=0$ for all $x \in U$.

For each $z \in \mathbb{R}^{n+1}$, the function $G_{z}$ defined by

$$
G_{z}(x)=\frac{1}{\sigma_{n}} \frac{\overline{z-x}}{|z-x|^{n+1}}
$$

for each $x \neq z$ is both left and right monogenic. Here the volume of the unit $n$-sphere in $\mathbb{R}^{n+1}$ has been denoted by $\sigma_{n}$ and we have used the identification of $\mathbb{R}^{n+1}$ with a subspace of $\mathbb{R}_{(n)}$ mentioned in Section 2 .

The function $G_{z}, z \in \mathbb{R}^{n+1}$ plays a special role in Clifford analysis. Suppose that $\Omega \subset \mathbb{R}^{n+1}$ is a bounded open set with smooth boundary $\partial \Omega$ and exterior unit normal $n(\omega)$ defined for all $\omega \in \partial \Omega$. For any left monogenic function $f$ defined in a neighbourhood $U$ of $\bar{\Omega}$, the Cauchy integral formula

$$
\int_{\partial \Omega} G_{\omega}(x) n(\omega) f(\omega) d \mu(\omega)=\left\{\begin{array}{lll}
f(x), & \text { if } & x \in \Omega \\
0, & \text { if } & x \in U \backslash \bar{\Omega}
\end{array}\right.
$$

is valid. Here $\mu$ is the surface measure of $\partial \Omega$. The result is proved in [3, Corollary 9.6]. If $g$ is right monogenic in $U$ then $\int_{\partial \Omega} g(\omega) n(\omega) f(\omega) d \mu(\omega)=0$ [3, Corollary 9.3].

The definition of monogenicity extends readily to other vector and operator valued functions. We remark here that, as in the case of vector valued analytic functions, a function is monogenic for the weak topology of a locally convex module $E_{(n)}$ if and only if it is monogenic for the original topology. Moreover, for a Banach space $E$, if $g: U \rightarrow$ $E_{(n)}$ is right monogenic and $f: U \rightarrow \mathbb{F}_{(n)}$ is left monogenic, then the function $\omega \mapsto$ $g(\omega) n(\omega) f(\omega), \omega \in \partial \Omega$, is Bochner $\mu$-integrable in $E_{(n)}$ and $\int_{\partial \Omega} g(\omega) n(\omega) f(\omega) d \mu(\omega)=$ 0 . In particular, this is valid in the case that $X$ is a Banach space and $E=\mathcal{L}(X)$ with the uniform operator norm. It follows from the principle of uniform boundedness and the Cauchy integral formula that an $\mathcal{L}(X)$-valued function is norm monogenic when it is monogenic for the weak or strong operator topologies.

\section{The Weyl functional calculus.}

Let $A_{1}, \ldots, A_{n}$ be bounded selfadjoint operators acting on a Hilbert space $H$ with inner product $\langle\cdot, \cdot\rangle$. The Weyl functional calculus $[\mathbf{9}, \mathbf{1}]$ is a means of forming functions $f_{\mathrm{W}}\left(A_{1}, \ldots, A_{n}\right)$ of the $n$-tuple $A=\left(A_{1}, \ldots, A_{n}\right)$ of operators. The operators $A_{1}, \ldots, A_{n}$ do not necessarily commute with each other, so there is no fundarnentally unique way of forming such functions. However, the Weyl functional calculus is determined by a few natural conditions, see $[1$, Theorem 2.4$]$. 
For every $\xi \in \mathbb{R}^{n},\langle A, \xi\rangle=\langle\xi, A\rangle$ denotes the selfadjoint operator $\sum_{j=1}^{n} A_{j} \xi_{j}$. The operator $e^{i\langle A, \xi\rangle}$ is therefore unitary for each $\xi \in \mathbb{R}^{n}$. The Fourier transform $\hat{f}$ of a function $f$ integrable over $\mathbb{R}^{n}$ is defined by $\hat{f}(\xi)=(2 \pi)^{-n / 2} \int_{\mathbb{R}^{n}} e^{-i\langle x, \xi\rangle} f(x) d x$ for all $\xi \in \mathbb{R}^{n}$. The integral $f_{W}(A)=(2 \pi)^{-n / 2} \int_{\mathbb{R}^{n}} e^{i\langle A, \xi\rangle} \hat{f}(\xi) d \xi$ is an operator valued Bochner integral for each function $f$ belonging to the space $\mathcal{S}\left(\mathbb{R}^{n}\right)$ of rapidly decreasing functions on $\mathbb{R}^{n}$. Then the mapping $f \mapsto f_{\mathrm{W}}(A)$, for all $f \in \mathcal{S}\left(\mathbb{R}^{n}\right)$ is the Weyl functional calculus for the $n$-tuple $A$ of selfadjoint operators.

There exists a unique operator valued distribution $\mathcal{W}_{A}: f \mapsto f_{\mathrm{W}}(A), f \in C^{\infty}\left(\mathbb{R}^{n}\right)$ defined over the test function space $C^{\infty}\left(\mathbb{R}^{n}\right)$ of all infinitely differentiable functions, such that the restriction of $\mathcal{W}_{A}$ to $\mathcal{S}\left(\mathbb{R}^{n}\right)$ is the Weyl calculus for $A$. The support of this distribution is contained in the closed unit ball in $\mathbb{R}^{n}$ centred at zero and with radius $\left(\sum_{j=1}^{n}\left\|A_{j}\right\|^{2}\right)^{1 / 2}[9$, Theorem 1$]$.

The set $\mathbb{R}^{n}$ is identified with the subspace $\left\{x \in \mathbb{R}^{n+1}: x_{0}=0\right\}$ of $\mathbb{R}^{n+1}$. Suppose that $U$ is an open neighbourhood of the support of $\mathcal{W}_{A}$ in $\mathbb{R}^{n}$. There is no harm if we also call the distribution $\mathcal{W}_{A}: f \mapsto f_{\mathrm{W}}(A)$ over $C^{\infty}(U)$ the Weyl functional calculus for $A$. The algebraic tensor product $\mathcal{W}_{A} \otimes I_{(n)}: C^{\infty}(V)_{(n)} \rightarrow \mathcal{L}(H)_{(n)}$ of $\mathcal{W}_{A}$ with the identity operator $I_{(n)}$ on $\mathbb{F}_{(n)}$ is also denoted just by $\mathcal{W}_{A}$. Here $V$ is an open neighbourhood of supp $\mathcal{W}_{A}$ in $\mathbb{R}^{n+1}$ and $C^{\infty}(V)_{(n)}$ is the locally convex module obtained by tensoring the locally convex space $C^{\infty}(V)$ with $\mathbb{F}_{(n)}$, as mentioned in Section 3. The mapping $\mathcal{W}_{A}: C^{\infty}(V) \rightarrow \mathcal{L}(H)$ is defined by applying $\mathcal{W}_{A}$ to the restriction of functions $f \in C^{\infty}(V)$ to the open subset $V \cap \mathbb{R}^{n}$ of $\mathbb{R}^{n}$. The map $\mathcal{W}_{A}: C^{\infty}(V)_{(n)} \rightarrow \mathcal{L}(H)_{(n)}$ is a module homomorphism. The symbols $\mathcal{W}_{A}(f)$ and $f_{\mathrm{W}}(A)$ are used interchangeably.

For any subset $G$ of $\mathbb{R}^{n+1}$, let $M\left(G, \mathbb{F}_{(n)}\right)$ be the collection of all $\mathbb{F}_{(n)}$-valued functions which are left monogenic in an open neighourhood of $G$ in $\mathbb{R}^{n+1}$. If $G$ is open in $\mathbb{R}^{n+1}$, then $M\left(G, \mathbb{F}_{(n)}\right)$ is given the compact-open topology (uniform convergence on every compact subset of $G)$. If $K$ is a compact subset of $\mathbb{R}^{n}$, then $M\left(K, \mathbb{F}_{(n)}\right)$ is the union of all spaces $M\left(U, \mathbb{F}_{(n)}\right)$, as $U$ ranges over the open sets in $\mathbb{R}^{n+1}$ containing $K$. The space $M\left(K, \mathbb{F}_{(n)}\right)$ is equipped with the inductive limit topology.

The support supp $\mathcal{W}_{A}$ of the distribution $\mathcal{W}_{A}$, which is independent of the particular meaning attached to it above, is a compact subset of $\mathbb{R}^{n}$. Let $U$ be an open neighbourhood of $K:=\operatorname{supp} \mathcal{W}_{A}$ in $\mathbb{R}^{n}$ and suppose that the function $f: U \rightarrow \mathbb{C}$ is analytic. Let $\tilde{f}$ be a left monogenic extension of $f$ to an open neighbourhood of $U$ in $\mathbb{R}^{n+1}$. Then according to the definition of $(\tilde{f})_{\mathrm{W}}(A)$, the equality $(\tilde{f})_{\mathrm{W}}(A)=f_{\mathrm{W}}(A) \otimes I_{(n)}$ is valid. The Weierstrass convergence theorem for monogenic functions [3, Theorem 9.11] ensures that the Weyl calculus $\mathcal{W}_{A}: M\left(K, \mathbb{F}_{(n)}\right) \rightarrow \mathcal{L}(H)_{(n)}$ is a continuous right 
module homomorphism. Then $M\left(K, \mathbb{F}_{(n)}\right)$ becomes a topological algebra under the C-K product [3, p113], and the closed subspace $M(K, \mathbb{F})$ of $M\left(K, \mathbb{F}_{(n)}\right)$ consisting of left monogenic extensions of $\mathbb{F}$-valued functions on $K$ is a commutative topological algebra. The topological algebra $M(K, \mathbb{F})$ is isomorphic, via monogenic extension, to the topological algebra $H(K, \mathbb{F})$ of $\mathbb{F}$-valued functions analytic in an open neighbourhood of $K$ with pointwise multiplication. The Weyl calculus is also continuous on this space.

Let $X$ be a Banach space. A sequence $\left\{f_{k}\right\}_{k=1}^{\infty}$ of $X$-valued functions $f_{k}: \Omega \rightarrow X$ is normally summable in $X$ if there exists a summable sequence $\left\{M_{k}\right\}_{k=1}^{\infty}$ of nonnegative real numbers $M_{k}$ such that $\left\|f_{k}(\omega)\right\| \leqslant M_{k}$, for all $\omega \in \Omega$ and all $k=1,2, \ldots$. Thus, a normally summable sequence $\left\{f_{k}\right\}_{k=1}^{\infty}$ of $X$-valued functions on $\Omega$ is absolutely and uniformly summable on $\Omega$.

Suppose that $f$ is an analytic $\mathbb{F}$-valued function defined on an open neighbourhood of zero in $\mathbb{R}^{n}$ and the Taylor series of $f$ is given by

$$
f(x)=\sum_{k=0}^{\infty} \frac{1}{k !} \sum_{l_{1}=1}^{n} \cdots \sum_{l_{k}=1}^{n} a_{l_{1} \ldots l_{k}} x_{l_{1}} \cdots x_{l_{k}}
$$

for all $x \in \mathbb{R}^{n}$ in a neighbourhood of zero. The coefficients $a_{l_{1} \ldots l_{k}}$ are assumed to be symmetric in $l_{1}, \ldots l_{k}$. Then the unique monogenic extension $\tilde{f}$ of $f$ is

$$
\tilde{f}(x)=\sum_{k=0}^{\infty}\left(\sum_{\left(l_{1}, \ldots, l_{k}\right)} a_{l_{1} \ldots l_{k}} V_{l_{1} \ldots l_{k}}(x)\right)
$$

for all $x$ belonging to some neighbourhood of zero in $\mathbb{R}^{n+1}$. Here, the sum $\sum_{\left(l_{1}, \ldots, l_{k}\right)} \ldots$ is over the set $\left\{1 \leqslant l_{1} \leqslant \ldots \leqslant l_{k} \leqslant n\right\}$, and for $\left(l_{1}, \ldots, l_{k}\right) \in\{1,2, \ldots, n\}^{k}$, the function $V_{l_{1} \ldots l_{k}}: \mathbb{R}^{n+1} \rightarrow \mathbb{F}_{(n)}$ is defined as follows. For each $j=1, \ldots, n$, the monogenic extension of the function $\mathbf{x}_{j}: x \mapsto x_{j}, x \in \mathbb{R}^{n}$ is given by $\mathbf{z}_{j}: x \mapsto x_{j} e_{0}-x_{0} e_{j}, x \in$ $\mathbb{R}^{n+1}$. Then $V_{0}(x)=e_{0}, x \in \mathbb{R}^{n+1}$ and $V_{l_{1} \ldots l_{k}}=1 / k ! \sum_{j_{1}, \ldots, j_{k}} \mathbf{z}_{j_{1}} \cdots \mathbf{z}_{j_{k}}$, where the sum is over all distinguishable permutations of $\left(l_{1}, \ldots, l_{k}\right)$, and products are in the sense of pointwise multiplication in $\mathbb{F}_{(n)}$. If $\tilde{f}$ is left monogenic in the open ball $B_{R}(0)$ of radius $R$ about zero in $\mathbb{R}^{n+1}$, then (2) converges normally in $B_{R}(0)[3, p .82]$.

Set $V_{l_{1} \ldots l_{k}}(A)=1 / k ! \sum_{j_{1}, \cdots, j_{k}} A_{j_{1}} \cdots A_{j_{k}}$ for the same ranges of indices. The product is understood as the composition of operators. Then, it follows easily from the observation of Nelson [8] and Anderson [1, Theorem 2.4 (c)], that the equality

$$
f_{\mathrm{W}}(A)=\sum_{k=0}^{\infty}\left(\sum_{\left(l_{1}, \ldots, l_{k}\right)} a_{l_{1} \ldots l_{k}} V_{l_{1} \ldots l_{k}}(A)\right)
$$


holds if (1) converges in a suitable neighbourhood of supp $\mathcal{W}_{A}$.

In the case in which the monogenic expansion of a function about a point does not converge over all of supp $\mathcal{W}_{A}$, the Cauchy integral formula is useful. Of course, this is the central idea of the Dunford-Riesz functional calculus for a single operator. Moreover, when $A$ is an $n$-tuple of operators acting on a Banach space and the Weyl functional calculus for $A$ is not defined - there is no exponential bound - the Cauchy integral formula can be used to define functions of the $n$-tuple $A$, see [5].

For any $z \in \mathbb{R}^{n+1}$ not belonging to supp $\mathcal{W}_{A}$, there exists an open neighbourhood $U_{z}$ of $\operatorname{supp} \mathcal{W}_{A}$ in $\mathbb{R}^{n+1}$, not containing $z$, such that the $\mathbb{F}_{(n)}$-valued function

$$
x \mapsto G_{z}(x)=\frac{1}{\sigma_{n}} \frac{\overline{z-x}}{|z-x|^{n+1}}, \text { for all } x \in U_{z},
$$

belongs to $C^{\infty}\left(U_{z}\right)_{(n)}$. Then $\mathcal{W}_{A}\left(G_{z}\right)=\left(G_{z}\right)_{\mathrm{W}}(A)$ may be viewed as an element of $\mathcal{L}(H)_{(n)}$.

EXAMPle 5.1. Let $n=3$ and consider the simplest non-commuting example of the Pauli matrices,

$$
J_{1}=\left(\begin{array}{cc}
0 & 1 \\
1 & 0
\end{array}\right), \quad J_{2}=\left(\begin{array}{cc}
0 & -i \\
i & 0
\end{array}\right), \quad J_{3}=\left(\begin{array}{cc}
1 & 0 \\
0 & -1
\end{array}\right),
$$

viewed as linear transformations acting on $H=\mathbb{C}^{2}$. Set $J=\left(J_{1}, J_{2}, J_{3}\right)$. A calculation $\left[\mathbf{1}\right.$, Theorem 4.1] shows that for all $f \in C^{\infty}\left(\mathbb{R}^{3}\right)$, the matrix $\mathcal{W}_{J}(f)$ is given by

$$
\mathcal{W}_{J}(f)=I \int_{S_{1}}(f+n \cdot \nabla f) d \mu_{1}+\int_{S_{1}} J \cdot \nabla f d \mu_{1} .
$$

Here $S_{t}=\left\{x \in \mathbb{R}^{3}:|x|=t\right\}$ is the sphere of radius $t>0$ centred at zero in $\mathbb{R}^{3}, \mu_{1}$ is the unit surface measure on $S_{1}$ and $n(x)$ is the outward unit normal at $x \in S_{1}$. Thus, $\operatorname{supp} \mathcal{W}_{J}=S_{1}$.

For all $\omega \in \mathbb{R}^{4}$ such that $\omega \notin S_{1} \subset \mathbb{R}^{3}, \mathcal{W}_{J}\left(G_{\omega}\right) \in \mathcal{L}\left(\mathbb{C}^{2}\right)_{(3)}$ is given by

$$
\mathcal{W}_{J}\left(G_{\omega}\right)=I \int_{S_{1}}\left(G_{\omega}+n \cdot \nabla G_{\omega}\right) d \mu_{1}+\int_{S_{1}} J \cdot \nabla G_{\omega} d \mu_{1} .
$$

Let $v_{1}, v_{2}$ be the standard basis vectors of $\mathbb{C}^{2}$. For each $x_{0} \in \mathbb{R}$, the function $(x, t) \mapsto$ $\mathcal{W}_{t J}\left(G_{\left(x+x_{0} e_{0}\right)}\right) v_{j}$ is the solution of the Weyl equation

$$
\partial_{t} u_{t}+J \cdot \nabla u_{t}=0, \quad t>0,
$$

with initial datum $u_{0}(x)=-v_{j} \otimes G_{0}\left(x+x_{0} e_{0}\right)=\sigma_{3}^{-1} v_{j} \otimes\left(x_{0} e_{0}-x\right) /\left|x+x_{0} e_{0}\right|^{4}$ for all $x \in \mathbb{R}^{3}, x+x_{0} e_{0} \neq 0$. The function $\omega \mapsto \mathcal{W}_{t J}\left(G_{\omega}\right) v_{j}$ is left and right monogenic on the set $\mathbb{R}^{4} \backslash S_{t}$. 
The following statements are formulated in a context more general than that of the Weyl functional calculus.

Suppose that $H$ is a Hilbert space over the field $\mathbb{F}$ and $T: C^{\infty}\left(\mathbb{R}^{n}\right) \rightarrow \mathcal{L}(H)$ is a distribution with compact support $K$. We use the same symbol $T$ to denote the map which sends the element $f=\sum_{S} f_{S} e_{S}$ of $C^{\infty}(K)_{(n)}$ to the element $\sum_{S} T\left(f_{S}\right) e_{S}$ of $\mathcal{L}(H)_{(n)}$, rather than the more descriptive notation $T \otimes I_{(n)}$. In particular, $T(f) \in$ $\mathcal{L}(H)_{(n)}$ is defined for all $f \in M\left(K, \mathbb{F}_{(n)}\right)$.

Proposition 5.2. Let $U$ be an open subset of $\mathbb{R}^{n+1}$ containing $K=\operatorname{supp} T$. Suppose that $z \mapsto F_{z}$ is a continuous map from $U \backslash K$ into $C^{\infty}(K)_{(n)}$. If for each open set $V$ with $\bar{V} \subset U \backslash K$, there exists a neighbourhood $N_{V}$ of $K$, such that for each $x \in N_{V}$, the $\mathbb{F}_{(n)}$-valued function $z \mapsto F_{z}(x)$ is left monogenic in $V$, then $z \mapsto T\left(F_{z}\right)$ is left monogenic in $U \backslash K$.

Proof: By Cauchy's theorem for monogenic functions [3, Theorem 9.6], for all intervals $I$ contained in $U \backslash K, \int_{\partial I} n(\omega) F_{\omega}(x) d \mu(\omega)=0$ for each $x$ belonging to some neighbourhood of $K$. The function $z \mapsto F_{z}, z \in U \backslash K$ is continuous, and so Bochner integrable in $C^{\infty}(K)_{(n)}$ on all boundaries $\partial I$ of intervals $I$ contained in $U \backslash K$. Moreover, the function $\int_{\partial I} n(\omega) F_{\omega} d \mu(\omega)$ belongs to $C^{\infty}(K)_{(n)}$ and vanishes in a neighbourhood of the support $K$ of $T$.

The distribution $T: C^{\infty}(K)_{(n)} \rightarrow \mathcal{L}(H)_{(n)}$ is a continuous linear map, so as observed in the introduction, the equalities

$$
\int_{\partial I} n(\omega) T\left(F_{\omega}\right) d \mu(\omega)=T\left(\int_{\partial I} n(\omega) F_{\omega} d \mu(\omega)\right)=0
$$

hold. By Morera's theorem for monogenic functions [3, Theorem 10.4], $z \mapsto T\left(F_{z}\right)$ is left monogenic in $U \backslash K$.

The same result holds for right monogenic functions.

Corollary 5.3. The $\mathcal{L}(H)_{(n)}$-valued function $z \mapsto \mathcal{W}_{A}\left(G_{z}\right)$ is left and right monogenic in $\mathbb{R}^{n+1} \backslash \operatorname{supp} \mathcal{W}_{A}$.

THEOREM 5.4. Let $T$ be an $\mathcal{L}(H)$-valued distribution with compact support. Let $\Omega$ be a bounded open neighbourhood of $\operatorname{supp} T$ in $\mathbb{R}^{n+1}$ with smooth boundary $\partial \Omega$ and exterior unit normal $n(\omega)$ defined for all $\omega \in \partial \Omega$. Let $\mu$ be the surface measure of $\Omega$.

Suppose that $f$ is left monogenic and $g$ is right monogenic in a neighbourhood of the closure $\bar{\Omega}=\Omega \cup \partial \Omega$ of $\Omega$. Then

$$
\begin{aligned}
& T(f)=\int_{\partial \Omega} T\left(G_{\omega}\right) n(\omega) f(\omega) d \mu(\omega), \\
& T(g)=\int_{\partial \Omega} g(\omega) n(\omega) T\left(G_{\omega}\right) d \mu(\omega) .
\end{aligned}
$$


ProOF: We consider only the case where $f$ is left monogenic. The case where $g$ is right monogenic is similar. The space $C^{\infty}(\Omega)_{(n)}$ of smooth $\mathbb{F}_{(n)}$-valued functions defined on $\Omega$ is a separable Fréchet space with the topology of uniform convergence of functions, and their derivatives, on compact subsets of $\Omega$. The continuous function $\omega \mapsto G_{\omega} n(\omega) f(\omega), \omega \in \partial \Omega$ takes its values in $C^{\infty}(\Omega)_{(n)}$ and satisfies $\int_{\partial \Omega} p\left(G_{\omega} n(\omega)\right)|f(\omega)| d \mu(\omega)<\infty$ for each continuous seminorm $p$ on $C^{\infty}(\Omega)_{(n)}$, that is, it is Bochner integrable in $C^{\infty}(\Omega)_{(n)}$.

By the Cauchy integral theorem mentioned in Section 4, the equality

$$
f(x)=\int_{\partial \Omega} G_{\omega}(x) n(\omega) f(\omega) d \mu(\omega)
$$

holds for all $x$ belonging to the open set $\Omega$. Combining this equation with the observation made in the Introduction, and the fact that the distribution $T$ defines a continuous linear map (denoted by the same symbol) from $C^{\infty}(\Omega)_{(n)}$ into the space $\mathcal{L}(H)_{(n)}$ with the uniform operator norm, it follows that the function $\omega \mapsto T\left(G_{\omega}\right) n(\omega) f(\omega), \omega \in \partial \Omega$ is Bochner integrable in the space $\mathcal{L}(H)_{(n)}$, with the uniform norm, and the equality

$$
T\left(\int_{\partial \Omega} G_{\omega} n(\omega) f(\omega) d \mu(\omega)\right)=\int_{\partial \Omega} T\left(G_{\omega}\right) n(\omega) f(\omega) d \mu(\omega)
$$

obtains. The stated equality $T(f)=\int_{\partial \Omega} T\left(G_{\omega}\right) n(\omega) f(\omega) d \mu(\omega)$ therefore holds.

CoRollary 5.5. Let $\Omega$ be a bounded open neighbourhood of supp $\mathcal{W}_{A}$ in $\mathbb{R}^{n+1}$ with smooth boundary $\partial \Omega$ and exterior unit normal $n(\omega)$ defined for all $\omega \in \partial \Omega$. Let $\mu$ be the surface measure of $\Omega$.

Suppose that $f$ is left monogenic and $g$ is right monogenic in a neighbourhood of the closure $\bar{\Omega}=\Omega \cup \partial \Omega$ of $\Omega$. Then

$$
\begin{aligned}
& f_{\mathrm{W}}(A)=\int_{\partial \Omega} \mathcal{W}_{A}\left(G_{\omega}\right) n(\omega) f(\omega) d \mu(\omega) \\
& g_{\mathrm{W}}(A)=\int_{\partial \Omega} g(\omega) n(\omega) \mathcal{W}_{A}\left(G_{\omega}\right) d \mu(\omega)
\end{aligned}
$$

We mention here that the extension of these results to $H$-valued functions is straightforward. First, if $f=\sum_{j=1}^{n} f_{j} h_{j}$ for monogenic functions $f_{j}$ and vectors $h_{j} \in H$, then $T(f)=\sum_{j} T\left(f_{j}\right) h_{j}$ and the above equality holds. In the limit, both sides of the equation converge because $C^{\infty}(\operatorname{supp} T) \otimes H$ is dense in $C^{\infty}(\operatorname{supp} T ; H)$. 


\section{The Monogenic Spectrum}

Let $A$ be an $n$-tuple of bounded selfadjoint operators acting on a Hilbert space $H$. For each $\omega \in \mathbb{R}^{n+1}$ such that $\omega \neq 0$, let

$$
G_{\omega}(x)=\sum_{k=0}^{\infty}\left(\sum_{\left(l_{1}, \ldots, l_{k}\right)} W_{l_{1} \ldots l_{k}}(\omega) V_{l_{1} \ldots l_{k}}(x)\right)
$$

be the monogenic power series expansion of $G_{\omega}$ in the region $|x|<|\omega|[3,11.4 \mathrm{pp} 77-$ 81]. Here $W_{l_{1} \ldots l_{k}}(\omega)$ is given for each $\omega \in \mathbb{R}^{n+1}, \omega \neq 0$ by $(-1)^{k} \partial_{\omega_{l_{1}}} \cdots \partial_{\omega_{l_{k}}} G_{\omega}(0)$ and $V_{l_{1} \ldots l_{k}}$ is defined as in Section 5 .

It follows from formula (3) that

$$
\left(G_{\omega}\right)_{\mathrm{W}}(A)=\sum_{k=0}^{\infty}\left(\sum_{\left(l_{1}, \ldots, l_{k}\right)} W_{l_{1} \ldots l_{k}}(\omega) V_{l_{1} \ldots l_{k}}(A)\right)
$$

for all $\omega \in \mathbb{R}^{n+1}$ such that $|\omega|>(1+\sqrt{2})\left\|\sum_{j=1}^{n} A_{j} e_{j}\right\|$. Formula (5) is adopted as a definition of the Cauchy kernel in [6, Definition 3.11]. The sum converges in $\mathcal{L}(H)_{(n)}$ because of the following result.

LemMa 6.1. The sum

$$
\sum_{k=0}^{\infty} \sum_{\left(l_{1}, \ldots, l_{k}\right)}\left|W_{l_{1} \ldots l_{k}}(\omega)\right|\left\|V_{l_{1} \ldots l_{k}}(A)\right\|
$$

converges uniformly for $|\omega| \geqslant R, \omega \in \mathbb{R}^{n+1}$, for each $R>(1+\sqrt{2})\left\|F \sum_{j=1}^{n} A_{j} e_{j}\right\|$.

Proof: The norm $\left\|V_{l_{1} \ldots l_{k}}(A)\right\|$ of $V_{l_{1} \ldots l_{k}}(A)$ is bounded by

$$
\frac{1}{k !} \sum_{j_{1}, \ldots, j_{k}}\left\|A_{j_{1}}\right\| \cdots\left\|A_{j_{k}}\right\|
$$

where the sum is over all distinguishable permutations of $\left(l_{1}, \ldots, l_{k}\right)$. Suppose that for each $j=1, \ldots, n$, the index $j$ appears exactly $k_{j}$ times in the $k$-tuple $\left(l_{1}, \ldots, l_{k}\right)$. Then $k=k_{1}+\cdots+k_{n}$ and there are $k ! /\left(k_{1} ! \cdots k_{n} !\right)$ distinguishable permutations of $\left(l_{1}, \ldots, l_{k}\right)$. Thus, $\left\|V_{l_{1} \ldots l_{k}}(A)\right\| \leqslant\left(1 /\left(k_{1} ! \cdots k_{n} !\right)\right)\left\|A_{1}\right\|^{k_{1}} \cdots\left\|A_{n}\right\|^{k_{n}}$. It suffices to show that for each $R>(1+\sqrt{2})\left\|\sum_{j=1}^{n} A_{j} e_{j}\right\|$, the sum

$$
\sum_{k_{1}, \ldots, k_{n}=0}^{\infty} \frac{1}{k_{1} ! \cdots k_{n} !}\left|\partial_{\omega_{1}}^{k_{1}} \cdots \partial_{\omega_{n}}^{k_{n}} G_{\omega}(0)\right|\left\|A_{1}\right\|^{k_{1}} \cdots\left\|A_{n}\right\|^{k_{n}}
$$


converges uniformly for all $|\omega| \geqslant R, \omega \in \mathbb{R}^{n+1}$. However, this follows from the normal convergence of the multiple power series

$$
\frac{1}{|y-x|^{n-1}}=\sum_{k=0}^{\infty} \frac{(-1)^{k}}{k !}\left\langle x, \nabla_{y}\right\rangle^{k} \frac{1}{|y|^{n-1}}
$$

for $|x|<(\sqrt{2}-1)|y|[3, \mathrm{p} .83]$ and the equality $G_{\omega}(x)=\left(1 / \sigma_{n}\right) \overline{D_{x}}\left(1 /|\omega-x|^{n-1}\right)$, valid for all $\omega \neq x$.

We know from Corollary 5.3 that the function defined by formula (5) for all $|\omega|>$ $(1+\sqrt{2})\left\|\sum_{j=1}^{n} A_{j} e_{j}\right\|$ is actually the restriction of an $\mathcal{L}(H)_{(n)}$-valued function monogenic in $\mathbb{R}^{n+1} \backslash \operatorname{supp} \mathcal{W}_{A}$. The question remains as to whether there is a larger open set on which this function has a monogenic extension.

The spectrum of a single operator $T$ is the set of 'singularities' of the resolvent function $\lambda \mapsto(\lambda I-T)^{-1}$. Similarly, the monogenic spectrum $\gamma(A)$ of the $n$-tuple $A$ of bounded selfadjoint operators is the complement of the largest open set $U \subset \mathbb{R}^{n+1}$ in which the function $\omega \mapsto\left(G_{\omega}\right)_{\mathrm{W}}(A)$ is the restriction of a monogenic function with domain $U$.

THEOREM 6.2. Let $A$ be an $n$-tuple of bounded selfadjoint operators acting on a Hilbert space $H$. Then $\gamma(A)=\operatorname{supp} \mathcal{W}_{A}$.

Proof: We have established in Corollary 5.3 that $\gamma(A) \subseteq \operatorname{supp} \mathcal{W}_{A}$. Let $x \in$ $\gamma(A)^{c}$, let $U \subset \gamma(A)^{c}$ be an open neighbourhood of $x$ in $\mathbb{R}^{n}$ and suppose that $\phi$ is a smooth function with compact support in $U$.

Let $g, h \in H$. A comparison with [3, Definition 27.6] shows that the $\mathbb{F}_{(n)}$-valued monogenic function $\omega \mapsto\left\langle\left(G_{\omega}\right)_{\mathrm{W}}(A) g, h\right\rangle, \omega \in \mathbb{R}^{n+1} \backslash \operatorname{supp} \mathcal{W}_{A}$, is actually the monogenic representation of the distribution $\left\langle\mathcal{W}_{A} g, h\right\rangle: f \mapsto\left\langle\mathcal{W}_{A}(f) g, h\right\rangle$, for all smooth $f$ defined in an open neighbourhood of $\operatorname{supp} \mathcal{W}_{A}$. Then $\left\langle\mathcal{W}_{A} g, h\right\rangle\left(G_{\omega}\right)=\left\langle\left(G_{\omega}\right)_{\mathrm{W}}(A) g, h\right\rangle$ and by [3, Theorem 27.7$]$,

$$
\left\langle\mathcal{W}_{A} g, h\right\rangle(\phi)=\lim _{y_{0} \rightarrow 0^{+}} \int_{U}\left[\left\langle\left(G_{y+y_{0} e_{0}}\right)_{\mathrm{W}}(A) g, h\right\rangle-\left\langle\left(G_{y-y_{0} e_{0}}\right)_{\mathrm{W}}(A) g, h\right\rangle\right] \phi(y) d y .
$$

Because $\omega \mapsto\left(G_{\omega}\right)_{\mathrm{W}}(A)$ is monogenic (hence continuous) for all $\omega$ in $U$, the limit is zero, that is, $\left\langle\mathcal{W}_{A} g, h\right\rangle(\phi)=0$ for all $g, h \in H$ and all smooth $\phi$ supported by $U$. Hence $x \in \operatorname{supp} \mathcal{W}_{A}^{c}$, as was to be proved.

REMARK. The significance of the Cauchy kernel $\omega \mapsto\left(G_{\omega}\right)_{\mathrm{W}}(A)$ is that it is the monogenic representation or 'Cauchy transform' of the distribution $\mathcal{W}_{A}$ off $\operatorname{supp} \mathcal{W}_{A}-$ the distribution $\mathcal{W}_{A}$ represents the 'boundary values' on $\mathbb{R}^{n}$ of the monogenic function $\omega \mapsto\left(G_{\omega}\right)_{\mathrm{W}}(A)$. 
EXAmPle 6.3. Let $A=\left(J_{3}, J_{1}\right)$. It follows by applying [1, Theorem 2.9 (a)] to Example 5.1 that the support of $\mathcal{W}_{A}$ is the closed unit disk $\mathbf{D} \subset \mathbb{R}^{2}$ centred at zero, so $\gamma(A)=\mathbf{D}$. An explicit calculation is given in [4, Example 2]. The Clifford spectrum $\sigma(A)$ of $[6$, Definition 3.1] is $\sigma(A)=\{(0,0)\}$.

\section{REFERENCES}

[1] R.F.V. Anderson, 'The Weyl functional calculus', J. Funct. Anal. 4 (1969), 240-267.

[2] R.F.V. Anderson, 'On the Weyl functional calculus', J. Funct. Anal. 6 (1970), 110-115.

[3] F. Brackx, R. Delanghe and F. Sommen, Clifford analysis, Research Notes in Mathematics 76 (Pitman, Boston, London, Melbourne, 1982).

[4] G. Greiner and W. Ricker, 'Commutativity of compact operators', Studia Math. 112 (1995), 109-125.

[5] B. Jefferies, A. McIntosh and J. Picton-Warlow, 'The monogenic functional calculus' (to appear).

[6] V.V. Kisil, 'Möbius transformations and monogenic functional calculus', Electron. Res. Announc. Amer. Math. Soc. 2 (1996), 26-33.

[7] A. McIntosh and A. Pryde, 'A functional calculus for several commuting operators', Indiana Univ. Math. J. 36 (1987), 421-439.

[8] E. Nelson, 'A functional calculus for non-commuting operators', in Functional analysis and related fields, (F.E. Browder, Editor), Proceedings of a conference in honour of Professor Marshal Stone, Univ. of Chicago, May 1968 (Springer-Verlag, Berlin, Heidelberg, New York, 1970), pp. 172-187.

[9] M.E. Taylor, 'Functions of several self-adjoint operators', Proc. Amer. Math. Soc. 19 (1968), 91-98.

School of Mathematics

The University of New South Wales

Sydney NSW 2052

Australia

e-mail: B.Jefferies@unsw.edu.au
School of Mathematics, Physics, Computing and Electronics

Macquarie University

Sydney NSW 2109

Australia

e-mail: alan@mpce.mq.edu.au 